\section{Can training schemes incorporate valuable out-of-hours experience to reflect patient need?}

We read with interest the College's report OP95, ${ }^{1}$ prompted by concerns over a lack of supervised trainee exposure to emergency psychiatric presentations, particularly out of hours. We carried out a retrospective study into the demands psychiatric presentations pose on both the liaison psychiatry service and the emergency department. The aim was to determine whether the liaison psychiatry service met the demand for out-of-hours presentations.

The study examined a total of 116 presentations over 2 months and covered 81 patients (46\% of whom re-presented at least once). The patients were between 15 and 68 years old; $54 \%$ were female and $46 \%$ were male. Self-harm was the most common cause for presentation (53\%), followed by suicidal ideation (37\%). Other complaints included hallucinations, anxiety and 'strange behaviour', with some patients presenting with a combination of the above. Of these presentations, 89 (77\%) occurred out of hours, most commonly on Sunday. Interestingly, although there was an overall higher presentation rate overnight, the peak presentation time frame was between $14: 00 \mathrm{~h}$ and $14: 59 \mathrm{~h}$

Out of the 55 referrals to psychiatry services, 40 (72\%) were made out of hours, meaning the day liaison psychiatry team received only 9 documented referrals. Since the vast majority of psychiatric presentations and psychiatry referrals from the general hospital emergency department occur out of hours, this reinforces the importance of trainees gaining adequate out-of-hours experience to learn to manage these complex patients safely. Increased exposure would allow trainees to develop competence in managing such complex situations and also develop the necessary expertise to supervise others. Of course, we must also acknowledge that this idea creates a catch-22 situation: an increase in junior trainee input would naturally create an increased demand for senior doctors to take up out-of-hours supervisory roles. The impact of this on 9 to 5 working, banding and recruitment could be considerable, and would require consultation and agreement from senior doctors.

Pauline L. Gammack, FY2 doctor, NHS Greater Glasgow and Clyde, UK and Rekha Hegde, consultant in old age psychiatry, Leverndale Hospital, Glasgow, UK, email: rekha.hegde@ggc.scot.nhs.uk

1 Royal College of Psychiatrists. Training Psychiatrists in Emergency and Out-Of-Hours Care: Report of the Emergency Psychiatry Training Taskforce (Occasional Paper OP95). Royal College of Psychiatrists, 2015.

doi: $10.1192 / \mathrm{pb} .39 .6 .316$

\section{Legal highs, NPS, head shop drugs? Whatever you call them, we need to know more about prevalence}

In his letter ${ }^{1}$ John Lally rightly highlights the ongoing issue of limited information on the important clinical topic of novel psychoactive substances (NPS) - also known as legal highs and head shop drugs - and their use by mental health patients. He refers to his prevalence study in community mental health services, which remains, to our knowledge, the only one of its kind. This knowledge gap chimes with the College Faculty of Addictions Psychiatry report on NPS ${ }^{2}$ pointing out that currently mental health services in the UK have no system-wide method to record psychological harm related to club drugs and NPS.

In an effort to estimate local NPS use prevalence rates in patients presenting to acute mental health services in North Devon, we undertook a small retrospective survey of 100 consecutive acute psychiatric presentations (50 crisis team and 50 in-patient admissions) in January and February 2015. The overall prevalence of NPS use was $8 \%$, a little lower than the $13 \%$ described by Lally in his community sample, and it was higher in the in-patient group (12\%) than the crisis team group (4\%). Based on patients' self-reports, Lally found that in $54 \%$ of his community patients the substance taken had an adverse effect on their mental state (mainly psychosis). In our acute setting, the supervising consultant psychiatrists felt that in the majority of cases $(n=7 / 8,87 \%)$ NPS use was clinically relevant to the clinical presentation. ICD-10 diagnoses of patients with acute presentation were also predominantly psychotic $(n=5 / 8,62.5 \%)$.

The locality service covers a large catchment area, with a well-dispersed population of about 150000 living in an area of 420 square miles. Of the seven people living locally, six had residential addresses within a mile of a shop known to be openly selling legal highs; the remaining lived within 2 miles of the shop. There were no people from towns without known legal high shops. This is of potential interest and relevance to any public health or local government interventions.

This was a small sample, with much more simplistic methodology than Lally's study, making any firm conclusions difficult. Given its retrospective nature, and reliance on individual's disclosure and clinician's documentation, our results are likely to be an underestimate of the true prevalence. However, we are aware of no other published record of NPS use prevalence rates in an acute psychiatric population.

Andrew P. Moore, consultant psychiatrist, Devon Partnership NHS Trust, Braunton, UK, email: andrew.moore7@nhs.net, and Elly Lesser, general practice trainee (GPST2), Devon Partnership NHS Trust, Barnstaple, UK.

1 Lally J. 'Legal highs'- what's in a name. BJPsych Bull 2015; 39: 206.

2 Bowden-Jones O, Fitch C, Hilton C, Lewis J, Ofori-Attah G. One New Drug a Week: Why Novel Psychoactive Substances and Club Drugs Need a Different Response from UK Treatment Providers (Faculty Report FR/AP/ 02). Royal College of Psychiatrists, 2014.

doi: 10.1192/pb.39.6.316a

\section{Plus ça change}

I must say I was deeply sceptical about the Bulletin comment, especially the assertion, 'Ward rounds have been taking place for decades; had they been purely detrimental they surely would have been junked years ago.' Maybe as someone who has mainly worked in psychotherapy and latterly as a community psychiatrist, I could be considered not qualified to comment, but the article took me back to my training with Dr Sidney Benjamin in Manchester in the early 1980s. He gave the example of videoing the exchange between himself and the patient in a separate room, with only the senior house officer ( $\mathrm{SHO}$ ) present to take verbatim notes of the consultation; the rest of the team could watch the interaction comfortably in another room. I think patients quite enjoyed 'being on TV'; it was somewhat nerve-wracking for the $\mathrm{SHO}$, as a perfect transcription was expected, but overall it was therapeutic for 
the patient and an excellent learning experience for the trainee. My occasional glimpses of intimidating ward rounds since as an observer have done nothing to convince me that Sidney Benjamin's format has been bettered.

Teresa J. Black, consultant psychiatrist, Black Country Partnership NHS Foundation Trust, Wolverhampton, UK, email: Teresa.Black@bcpft.nhs.uk

1 Bulletin comment: In praise of the psychiatric ward round. BJPsych Bull 2015; 39: 260 .

doi: $10.1192 / \mathrm{pb} .39 .6 .316 \mathrm{~b}$

\section{The GMC review of fitness to practise investigations and its impact on doctors}

The fact that doctors have a higher suicide rate than the general population is of great concern. ${ }^{1}$ Given the death of several doctors while under the General Medical Council (GMC) fitness to practise procedures, the internal review and report on this matter is very welcome. ${ }^{2}$ Contact with the GMC can result in significant anxiety for doctors.

What did the report reveal? The imbalance in resources for support in areas without access to services such as the Practitioner Health Programme (PHP) and Mednet was noted, and the suggestion of a national support service to provide doctors with confidential treatment is welcome. However, funding this service by increasing GMC fees could be perceived as a conflict of interest. Offers of support from the GMC are likely to be viewed at best in an ambivalent or distrustful light. Indeed, despite being run by the British Medical Association (BMA), doctors were concerned about revealing information to a $\mathrm{GMC}$-funded helpline. ${ }^{2}$ Doctors under investigation are likely to be even more reluctant to discuss issues with someone from the GMC as they may perceive that any information shared could have an impact on the investigation, potentially resulting in them struggling to access the support that they desperately need and a lack of engagement. In this respect, a PHP-like service has the advantage of being considered confidential and accessible. It was disappointing that the report mentioned neither the Royal College of Psychiatrists nor their Psychiatrists' Support Service (www.rcpsych.ac.uk/pss), established in 2007 to allow psychiatrists to talk, via telephone, to another psychiatrist and receive advice and signposting, a unique provision among medical Royal Colleges.
Doctors undergoing investigation often feel a sense of accusation and blame. Indeed, in 2007 the GMC changed the burden of proof requirement to follow the civil standard, 'on the balance of probabilities', rather than the criminal standard, 'beyond all reasonable doubt', which may well lead to doctors feeling 'guilty until proven innocent'. ${ }^{3}$ The suggestion that doctors consider themselves 'innocent until proven guilty' is welcome; however, the report offers no suggestions as to how this could be achieved. It is worrying that the report did not mention any actions taken by the GMC in the six instances where doctors were known to be at risk of suicide. There was no indication that risk assessments were performed for these doctors, when psychiatric input was likely to have been beneficial. As most health referrals have a mental health component, it is vital to consider that doctors undergoing investigation may be unwell and the benefits of psychiatric expertise should be recognised. We echo the Faculty of Occupational Medicine's disappointment that the report failed to reference existing competencies for treating doctors effectively. 4

Declaration of interest: All authors are doctor advisors for the Psychiatrists' Support Service, Royal College of Psychiatrists. This article represents our personal view and not that of the College.

Elena Baker-Glenn, ST6 in general adult and old age psychiatry, Cambridgeshire and Peterborough NHS Foundation Trust, Cambridge, UK, email: elenabakerglenn@yahoo.co.uk, Jane Marshall, consultant psychiatrist, South London and Maudsley NHS Foundation Trust, London, UK and Ricky Caplan, honorary consultant psychiatrist, Greater Glasgow and Clyde Health Board, Glasgow, UK.

1 Meltzer H, Griffiths C, Brock A, Rooney C, Jenkins R. Patterns of suicide by occupation in England and Wales: 2001-2005. Br J Psychiatry 2008; 193: $73-6$.

2 Horsfall S. Doctors Who Commit Suicide while Under GMC Fitness to Practise Investigation. General Medical Council, 2014.

3 Dyer C. GMC approves change in the standard of proof. BMJ 2007; 335 1230-1.

4 Faculty of Occupational Medicine of the Royal College of Physicians. FOM response to GMC-commissioned report into doctors who commit suicide while under GMC fitness to practise investigations. Royal College of Physicians, 2014. Available at: http://www.fom.ac.uk/ press-releases/fom-response-to-gmc-commissioned-report-intodoctors-who-commit-suicide-while-under-gmc-fitness-to-practiseinvestigations.

doi: 10.1192/pb.39.6.317 